\title{
Reliable Computation of Binary Parameters in Activity Coefficient Models for Liquid-Liquid Equilibrium
}

Luke D. Simoni, Youdong Lin, Joan F. Brennecke and Mark A. Stadtherr*

Department of Chemical and Biomolecular Engineering University of Notre Dame, Notre Dame, IN 46556, USA

May 4, 2007

*Author to whom all correspondence should be addressed. Phone: (574) 631-9318; Fax: (574) 631-8366; E-mail: markst@nd.edu 


\begin{abstract}
A method based on interval analysis is presented for determining parameter values from mutual solubility data in two-parameter activity coefficient models for liquid-liquid equilibrium. The method is mathematically and computationally guaranteed to locate all sets of parameter values corresponding to stable phase equilibria. The technique is demonstrated with examples using the NRTL and electrolyte-NRTL (eNRTL) models. In two of the NRTL examples, results are found that contradict previous work. In the eNRTL examples, binary systems of an ionic liquid and an alcohol are considered. This appears to be the first time that a method for parameter estimation in the eNRTL model from binary LLE data (mutual solubility) has been presented.
\end{abstract}




\section{Introduction}

Excess Gibbs energy models, often expressed in terms of the equivalent activity coefficient, are widely used in the modeling of liquid-liquid equilibrium. For modeling binary systems, the models used typically have two temperature-dependent binary parameters that must be determined from experimental data. There are a wide variety of such two-parameter models, including the UNIQUAC, NRTL (with fixed nonrandomness parameter), electrolyte-NRTL (eNRTL), van Laar and Margules (three suffix) models. Values of the binary parameters can be determined directly from mutual solubility data at a given temperature [1, 2]. The equal activity conditions for liquid-liquid equilibrium provide two equations that, given the experimental phase compositions, can be solved directly for the two binary parameter values needed to fit the mutual solubility data, thereby providing an exact fit to the experimental results. While this approach is widely practiced $[3,4]$, and simple in concept, there are computational difficulties that may arise and that must be addressed. One difficulty is that the nonlinear equation system to be solved for the binary parameters has an unknown number of solutions. There may be one solution, no solution or, for some models, notably NRTL and its variants, even multiple solutions. A technique is needed for finding all solutions with certainty, or showing rigorously that there are none. Another difficulty is that equal activity is only a necessary, but not sufficient, condition for equilibrium. This means that some solutions for the binary parameters may correspond to states that are not stable (unstable or metastable). Thus, a rigorous phase stability test is needed to verify whether solutions for

the parameters represent stable equilibrium states. However, phase stability analysis is itself 
well known to be a challenging computational problem, as it requires the rigorous solution of a nonlinear and nonconvex global minimization problem.

In this paper, we describe how both of these difficulties can be dealt with using strategies based on interval analysis. The method described will find all solutions to the equal activity conditions within specified parameter intervals (which may be arbitrarily large) and do so with mathematical and computational certainty, and it will also rigorously solve the phase stability problem, again with certainty. Since the solution of the phase stability problem for excess Gibbs energy models using interval methods has already been described $[5,6]$, we will focus here on the problem of computing all solutions for the binary parameters from the equal activity conditions, a problem that has not been previously addressed using the interval approach. The problem formulation is presented in the next section, along with a discussion of earlier work. This is followed by a brief summary of the interval method to be used. Then, several examples are presented to demonstrate the technique. For these examples we will use the NRTL and eNRTL models (with fixed nonrandomness parameter); however, the methodology can be applied in connection with any two-parameter activity coefficient model for liquid-liquid equilibrium.

\section{Problem Formulation}

Consider liquid-liquid equilibrium in a binary system at fixed temperature and pressure. For this case, the necessary and sufficient condition for equilibrium is that the total Gibbs energy be at a global minimum. The first-order optimality conditions on the Gibbs energy 
lead to the familiar equal activity conditions,

$$
a_{i}^{\mathrm{I}}=a_{i}^{\mathrm{II}}, \quad i=1,2,
$$

stating the equality of activities of each component (1 and 2 ) in each phase (I and II). As noted above, this is a necessary but not sufficient condition for equilibrium. Given an activity coefficient model, expressed in terms of mole fractions, $x_{1}$ and $x_{2}=1-x_{1}$, and involving two binary parameters, $\theta_{12}$ and $\theta_{21}$, the equal activity conditions can be expressed as

$$
x_{i}^{\mathrm{I}} \gamma_{i}^{\mathrm{I}}\left(x_{1}^{\mathrm{I}}, x_{2}^{\mathrm{I}}, \theta_{12}, \theta_{21}\right)=x_{i}^{\mathrm{II}} \gamma_{i}^{\mathrm{II}}\left(x_{1}^{\mathrm{II}}, x_{2}^{\mathrm{II}}, \theta_{12}, \theta_{21}\right), \quad i=1,2
$$

Substituting in experimental values for the mole fractions in each phase results in the $2 \times 2$ system of nonlinear equations

$$
x_{i, \exp }^{\mathrm{I}} \gamma_{i}^{\mathrm{I}}\left(x_{1, \exp }^{\mathrm{I}}, x_{2, \exp }^{\mathrm{I}}, \theta_{12}, \theta_{21}\right)=x_{i, \exp }^{\mathrm{II}} \gamma_{i}^{\mathrm{II}}\left(x_{1, \exp }^{\mathrm{II}}, x_{2, \exp }^{\mathrm{II}}, \theta_{12}, \theta_{21}\right), \quad i=1,2,
$$

which can in principle be solved for the parameters $\theta_{12}$ and $\theta_{21}$.

As discussed above, a significant difficulty is that the number of solutions to Eq. (3) is not known a priori. Thus, standard local methods for nonlinear equation solving are inadequate. In many cases, use of local methods with different initial guesses will be an effective approach. However, in general, multistart approaches are not completely reliable, since the number of solutions being sought is not known, and so it cannot be known when to stop trying different starting points. Strategies such as homotopy/continuation also provide the capability for locating multiple solutions, but still provide no guarantees that all solutions will be found. We will demonstrate here an approach for solving Eq. (3) based on interval mathematics, in particular an interval-Newton method. This method provides the capability 
of finding, with complete certainty, all solutions for the binary parameters, $\theta_{12}$ and $\theta_{21}$, within specified search intervals.

Since Eq. (3) is not a sufficient condition for phase equilibrium, once a solution for the binary parameters is found it must be checked to determine whether or not it actually corresponds to a stable equilibrium state. The determination of phase stability is generally based on the concept of tangent plane analysis [7]. On a Gibbs energy versus composition surface, phases in equilibrium will share the same tangent plane, with the equilibrium compositions corresponding to the points of tangency. For this to be a stable equilibrium, the tangent plane must not cross (go above) the Gibbs energy surface. To implement a stability test, a standard approach [8] is to consider the "tangent plane distance" function, that is the distance of the Gibbs energy surface above the tangent plane. If this is ever negative, the state is not stable. To prove stability, it must be shown that the global minimum of the tangent plane distance function is zero, corresponding to the points of tangency. This is a challenging problem since there are often multiple local minima in the tangent plane distance. To solve this global optimization problem rigorously, we will also use an approach $[5,6]$ based on an interval-Newton method, which can find the global minimum in the tangent plane distance with complete certainty. For additional details on the formulation and solution of the phase stability problem, see Tessier et al. [6]. The approach used here differs only in that a somewhat more efficient interval-Newton algorithm is used. 


\section{$2.1 \quad$ NRTL}

In the examples considered below the NRTL model is used. This is a model for the excess Gibbs energy $g^{E} / R T$ and is given in Appendix A for a binary system. The nonrandomness parameter $\alpha$ is frequently taken to be fixed when modeling liquid-liquid equilibrium, and this is the case in the examples studied below. The binary parameters that must be determined from experimental data are then $\theta_{12}=\Delta g_{12}=R T \tau_{12}$ and $\theta_{21}=\Delta g_{21}=R T \tau_{21}$. For purposes of phase stability analysis, an expression for the Gibbs energy versus composition surface is needed. If the reference states are taken to be pure liquids 1 and 2 at system temperature and pressure, then the needed expression is given by the Gibbs energy of mixing

$$
\frac{g^{\mathrm{M}}}{R T}=x_{1} \ln x_{1}+x_{2} \ln x_{2}+\frac{g^{\mathrm{E}}}{R T} .
$$

The activity coefficient expresstions given by Eqs (A.2-A.3) are used in connection with Eq. (3) to obtain the system of equations to be solved for the NRTL interaction parameters $\theta_{12}=\Delta g_{12}$ and $\theta_{21}=\Delta g_{21}$. This equation system is usually solved [2, 3, 9] using standard local methods, such as Newton's method, but is well known to frequently have multiple solutions [9]. Multiple solutions can be found using local methods and trying multiple starting points; however, this approach is not guaranteed to find all the solutions, and can lead to incorrect solutions, as shown in Section 4.2. One approach to determining all solutions is to attempt first to determine the number of solutions, as described by Mattelin and Verhoeye [10]. However, this approach is not always reliable, as shown in Section 4.3. Another approach is described by Jacq and Asselineau [11], who reformulated the equation system in terms of the new variables $t_{1}=\tanh \left(\alpha \Delta g_{21} / 2 R T\right)$ and $t_{2}=\tanh \left(\alpha \Delta g_{12} / 2 R T\right)$. 
The entire parameter space can then be covered by $t_{1} \in[-1,1]$ and $t_{2} \in[-1,1]$. Solutions are sought using a "sweeping" method in which one tries to follow the path of one equation in the parameter space, while checking for intersections with the other equation. Since the path tracking requires that some finite step size be used, there is no guarantee that all solutions (intersections) will be found. We will demonstrate here the use of an interval-Newton method that will guarantee that all solutions are found.

\subsection{Electrolyte-NRTL}

In some of the example problems, the electrolyte-NRTL (eNRTL) model is used, and applied to LLE in binary liquid salt (1)/solvent (2) systems. This model assumes complete dissociation of the salt, as described by

$$
\text { Salt } \longrightarrow \nu_{+}(\text {Cation })^{z_{+}}+\nu_{-}(\text {Anion })^{z_{-}}
$$

where $z_{j}$ is the valency associated with ion $j$. As in the case of the non-electrolyte case, parameter estimation can be done by using mutual solubility data and solving the equal activity condition. Equal activity is most conveniently expressed in terms of the actual mole fractions, $y_{+}, y_{-}$, and $y_{2}=1-y_{+}-y_{-}$, of cation, anion and solvent, respectively, and by using mean ionic quantities for the salt (component 1). For some quantity $\xi$, the corresponding

mean ionic quantity for the salt is given by $\xi_{ \pm}=\left(\xi_{+}^{\nu_{+}} \xi_{-}^{\nu_{-}}\right)^{1 / \nu}$ with $\nu=\nu_{+}+\nu_{-}[12]$. In these terms, the equal activity condition of Eq. (2) can be restated as [13]

$$
y_{i}^{\mathrm{I}} \gamma_{i}^{\mathrm{I}}\left(y_{ \pm}^{\mathrm{I}}, y_{2}^{\mathrm{I}}, \theta_{12}, \theta_{21}\right)=y_{i}^{\mathrm{II}} \gamma_{i}^{\mathrm{II}}\left(y_{ \pm}^{\mathrm{II}}, y_{2}^{\mathrm{II}}, \theta_{12}, \theta_{21}\right), \quad i= \pm, 2
$$


Using the experimental mutual solubility data, we then obtain the $2 \times 2$ equation system

$$
y_{i, \exp }^{\mathrm{I}} \gamma_{i}^{\mathrm{I}}\left(y_{ \pm, \exp }^{\mathrm{I}}, y_{2, \exp }^{\mathrm{I}}, \theta_{12}, \theta_{21}\right)=y_{i, \exp }^{\mathrm{II}} \gamma_{i}^{\mathrm{II}}\left(y_{ \pm, \exp }^{\mathrm{II}}, y_{2, \exp }^{\mathrm{II}}, \theta_{12}, \theta_{21}\right), \quad i= \pm, 2
$$

to be solved for $\theta_{12}$ and $\theta_{21}$. The actual mole fractions are related to the observable mole fractions, $x_{1}$ and $x_{2}=1-x_{1}$, by

$$
\begin{aligned}
& y_{ \pm}=\frac{\nu_{ \pm} x_{1}}{(\nu-1) x_{1}+1} \\
& y_{2}=\frac{x_{2}}{(\nu-1) x_{1}+1} .
\end{aligned}
$$

The eNRTL model is an excess Gibbs energy model and is given in Appendix B for a binary system. Note that we have renormalized the model $[14,15]$ relative to a symmetric reference state (pure dissociated liquid 1 and pure liquid 2, both at system temperature and pressure). Again the nonrandomness parameter $\alpha$ is taken to be fixed, and the binary parameters that must be estimated are $\theta_{12}=\Delta g_{12}=R T \tau_{12}$ and $\theta_{21}=\Delta g_{21}=R T \tau_{21}$. The value to be used for the closest approach parameter $\rho$ will discussed in Section 4 . For purposes of phase stability analysis, the Gibbs energy versus composition surface is given by

$$
\frac{g^{M}}{R T}=\frac{\nu}{\nu_{ \pm}} y_{ \pm} \ln \left(\frac{\nu}{\nu_{ \pm}} y_{ \pm}\right)+y_{2} \ln \left(y_{2}\right)+\frac{g^{E}}{R T}
$$

To determine the binary parameters $\theta_{12}$ and $\theta_{21}$, we will solve the equal activity conditions expressed by Eq. (7). For this purpose, expressions for the activity coefficients are needed, and these are provided by Eqs. (B.6-B.10). Note that since all of the examples below involve 1:1 electrolytes, activity coefficient expressions are provided in Appendix B for this case only. 
In Section 4, we apply eNRTL to the case of LLE in binary solutions of ionic liquids (component 1) and alcohols (component 2), and solve the parameter estimation problem outlined above. This appears to be the first time that this particular parameter estimation problem has been addressed. The eNRTL model has been used before primarily in the correlation of activity coefficient and vapor pressure depression data for electrolytic systems (e.g., $[16,17,18])$ and when applied to LLE problems has been in the context of multicomponent systems, such as phase splitting with salt in a mixture of solvents (e.g., [13, 19]). In these cases, the parameter estimation problem is much different from the problem described here and requires the optimization of some goodness of fit criterion (which can also be done rigorously using interval methods [18]).

\section{Reliable Parameter Estimation Method}

For solving the $2 \times 2$ nonlinear systems given by Eq. (3) or Eq. (7) for the binary parameter values $\theta_{12}$ and $\theta_{21}$, we use a method based on interval mathematics, in particular an interval-Newton approach combined with generalized bisection (IN/GB). This is a deterministic technique that provides a mathematical and computational guarantee that all the solutions to the relevant equation system are found. For general background on interval mathematics, including interval arithmetic, computations with intervals, and intervalNewton methods, there are several good sources [20, 21, 22]. Details of the basic IN/GB algorithm employed here are given by Schnepper and Stadtherr [23], with important enhancements described by Gau and Stadtherr [24] and Lin and Stadtherr [25]. 
An important feature of this approach is that, unlike standard methods for nonlinear equation solving that require a point initialization, the IN/GB methodology requires only an initial interval, and this interval can be sufficiently large to enclose all physically reasonable results. The initial parameter search intervals used are given in Section 4. Intervals are searched for solutions using a powerful root inclusion test based on the interval-Newton method. This method can determine with mathematical certainty if an interval contains no solution or if it contains a unique solution. If it cannot be proven that that interval contains no solution or a unique solution, then the interval is bisected and the root inclusion test is applied eventually to each subinterval. Once an interval proven to contain a unique solution has been found, that solution can be found by either 1) continuing interval-Newton iterations, which will converge quadratically to a tight interval enclosure of the solution, or 2) switching to a routine local Newton method, which will converge to a point approximation of the solution starting from any point in the interval. On completion, the IN/GB algorithm will have determined tight enclosures or point approximations for all the solutions to Eq. (3) or Eq. (7).

The overall method for determining the binary parameter values can be summarized as follows:

1. Select initial search intervals for the parameters.

2. Use the interval-Newton approach to solve the equal activity conditions, obtaining all possible solutions for the parameters within the specified search intervals.

3. Test each solution to determine if it corresponds to a stable equilibrium state, again 
using an interval-Newton approach [6] to ensure correctness. Discard any solutions that do not represent stable states.

4. Examine the suitability of the remaining parameter solutions. There are various reasons that a solution might be considered unsuitable. Two such reasons are discussed in detail by Heidemann and Mandhane [9] and are: 1) The case in which the parameters give a $g^{\mathrm{M}}$ model showing more than one miscibility gap. Though theoretically possible, this type of behavior is rarely observed. 2) The case in which one or both of the parameter values are large negative numbers. This leads to values of $G_{12}$ and/or $G_{21}$ that are extremely large, resulting in a highly distorted and unrealistic Gibbs energy surface whose slope changes sharply near $x_{1}=0$ and/or $x_{1}=1$, but which is nearly linear elsewhere. Sørensen and Arlt [3] consider as unsuitable any parameter solution that gives a $g^{\mathrm{M}}$ model having multiple composition solutions to the equal activity equation (i.e., multiple lines bitangent to the Gibbs energy surface). This case (and the case of multiple miscibility gaps) can be characterized as having a $g^{\mathrm{M}}$ versus $x_{1}$ curve with more than two inflection points. A rationale for considering this case unsuitable is that if these parameter values are used, and the equal activity conditions have multiple composition solutions, then phase equilibrium problems based on this model will be more difficult to solve. However, since today there are interval-based computational methods (e.g., $[6,26])$ for easily dealing with this situation in computing phase stability and equilibrium, it is really not necessary to consider this a case of unsuitable parameters. 
By beginning with an arbitrarily large initial interval in Step 1, and by finding all possible parameter solutions in Step 2, we ensure that the most suitable set(s) of parameter values will remain after Steps 3 and 4.

\section{Results and Discussion}

In this section, we provide several examples demonstrating the use of the interval method for computing binary parameters from mutual solubility data in modeling liquid-liquid equilibrium. In the first three examples, only the NRTL model is used, and in two of these examples results are found that contradict previous work. The final three examples involve modeling of binary LLE in ionic liquid (IL) systems, using both NRTL and eNRTL. Using the parameter estimation method described here, our colleagues Crosthwaite et al. [27] have modeled several complete phase diagrams for IL-alcohol systems, and have shown that it is possible to correlate binary LLE data for these systems using NRTL with binary parameters having a linear temperature dependence. However, it does not appear that NRTL is a good predictive model in this context [28]. Thus, we are also interested in the use of the eNRTL model, and present here our parameter estimation strategy for this model.

In the examples that follow, we will use the arbitrarily large initial search intervals

$\theta_{12}=\Delta g_{12} \in\left[-1 \times 10^{6}, 1 \times 10^{6}\right] \mathrm{J} / \mathrm{mol}$ and $\theta_{21}=\Delta g_{21} \in\left[-1 \times 10^{6}, 1 \times 10^{6}\right] \mathrm{J} / \mathrm{mol}$. Of course, it is not really necessary to use such a large initial search interval, especially since, as discussed above, parameters that are large negative numbers may not be suitable. However, since it may not always be clear where to draw the line on what is too large, it is easiest to just 
use such an arbitrarily large initial interval, and to then examine all solutions and discard those that are unsuitable. All computations were done on a workstation running Linux with a $3.2 \mathrm{GHz}$ Intel Pentium $4 \mathrm{CPU}$. Computation times were on the order of seconds for problems involving NRTL, and on the order of minutes for problems involving eNRTL.

\section{$4.1 \quad n$-Octanol and Water}

This example demonstrates the routine use of the parameter estimation procedure described above. Consider a mixture of $n$-octanol (1) and water (2) at $T=313.15 \mathrm{~K}$ and atmospheric pressure. Based on smoothed experimental data from Sørensen and Arlt [3], the observed liquid-phase compositions at equilibrium are $x_{1, \exp }^{\mathrm{I}}=0.000144$ and $x_{1, \exp }^{\mathrm{II}}=0.7530$. Binary NRTL parameters are sought for the case of $\alpha=0.2$. Applying the interval method to the solution of Eq. (3) with this data yields four parameter solutions, as listed in Table 1 in terms of $\Delta g_{12}$ and $\Delta g_{21}$ as well as the equivalent $\tau_{12}$ and $\tau_{21}$.

The four parameter solutions found are now checked to see if they correspond to stable phase equilibrium. This is done rigorously using an interval-based global optimization method [6], as discussed above. Two of the parameter solutions (3 and 4) are found not to represent stable phase equilibrium, and thus they can be discarded. Examination of the $g^{\mathrm{M}}$ versus $x_{1}$ function for the remaining solutions shows that solution 2 represents a case with two immiscibility gaps, and so it is not a suitable solution. Solution 1 has only two inflection points in the $g^{\mathrm{M}}$ versus $x_{1}$ curve, and so it is the most suitable solution. Solution 1 is the solution given by Sørensen and Arlt [3]. 


\section{$4.2 n$-Butanol and Water}

Consider a mixture of $n$-butanol (1) and water (2) at $T=363.15 \mathrm{~K}$ and atmospheric pressure. Liquid-liquid phase equilibrium is observed experimentally with phase compositions $x_{1, \exp }^{\mathrm{I}}=0.020150$ and $x_{1, \exp }^{\mathrm{II}}=0.35970$. Heidemann and Mandhane [9] modeled this system using NRTL with $\alpha=0.4$. They obtained three solutions for the binary parameters, as shown in Table 2, in terms of $\tau_{12}$ and $\tau_{21}$. Applying the interval method to this system, we find only two solutions, as also shown in Table 2. The extra solution found by Heidemann and Mandhane [9] is well within the search space used by the interval method, so clearly is a spurious solution resulting from numerical difficulties in the local method used to solve Eq. (3). This can also be verified by direct substitution of solution 3 into the equal activity conditions, and is most easily seen when equal activity is expressed in terms of $\ln x_{i}$ and $\ln \gamma_{i}$ (by taking the logarithm of both sides of Eq. (3)). Both of the valid parameter solutions correspond to stable phase equilibria. Solution 1 is the preferred solution, since solution 2 leads to a model that predicts two immiscibility gaps.

\subsection{1, 4-Dioxane and 1,2,3-Propanetriol}

Consider a mixture of 1,4-dioxane (1) and 1,2,3-propanetriol (2) at $T=298.15 \mathrm{~K}$ and atmospheric pressure. Liquid-liquid phase equilibrium is observed experimentally with phase

compositions $x_{1, \exp }^{\mathrm{I}}=0.2078$ and $x_{1, \exp }^{\mathrm{II}}=0.9934$. Mattelin and Verhoeye [10] modeled this system using NRTL with various values of $\alpha$. We will focus first on the case of $\alpha=0.15$. For this case, Mattelin and Verhoeye [10] obtained six solutions for the binary parameters, 
which are reported graphically without giving exact numerical values. Applying the interval method, we find only four solutions, as shown in Table 3 in terms of $\tau_{12}$ and $\tau_{21}$. The extra solutions found by Mattelin and Verhoeye [10] are well within the search space used by the interval method. Again, it appears that numerical difficulties in the use of local methods has led to spurious solutions. Two of the four parameter solutions correspond to stable phase equilibria. However, one parameter in solution 2 is a large negative number and results in a highly distorted Gibbs energy surface. Thus solution 1 is the preferred parameter estimate.

Looking at the case of $\alpha=0.25$, the interval method finds two parameter solutions, as indicated in Table 3. For this case, Mattelin and Verhoeye [10] obtained three solutions. In Table 4 , we summarize results for the several different values of $\alpha$ considered by Mattelin and Verhoeye [10], showing the number of solutions that they found versus the number of solutions found using the interval method. It can be seen that there are many cases in which extra and incorrect solutions were found by Mattelin and Verhoeye [10]. The fact that we always find an even number of solutions is consistent with Jacq and Asselineau [11], who argue that, in general, there must be an even number of parameter solutions for NRTL. Theoretically, there are specific values of $\alpha$ for which there are an odd number of solutions. For example, on this problem, as $\alpha$ is increased from 0.20 to 0.25 , two of the four solutions will approach each other, and at some specific value of $\alpha$ will coincide, leaving only three distinct solutions. Beyond this critical value of $\alpha$ there are then only two solutions. Since it is unlikely that such a critical value of $\alpha$ will be specified, in practice an even number of solutions should exist when using NRTL. Whether all of these are actually found would 
depend on the intial search interval.

\section{$4.4 \quad[$ bmpy $]\left[\mathrm{Tf}_{2} \mathrm{~N}\right]$ and $n$-Hexanol}

Consider a mixture of 1-n-butyl-3-methylpyridinium bis(trifluoromethylsulfonyl)imide ([bmpy] $\left.\left[\mathrm{Tf}_{2} \mathrm{~N}\right]\right)(1)$ and $n$-hexanol $(2)$ at $T=321 \mathrm{~K}$ and atmospheric pressure. The experimental liquid-phase compositions, based on the cloud point data of Crosthwaite et al. [27], are $x_{1, \exp }^{\mathrm{I}}=0.0206$ and $x_{1, \exp }^{\mathrm{II}}=0.4450$. In terms of the actual mean ionic mole fraction,

these are $y_{ \pm, \exp }^{\mathrm{I}}=x_{1, \exp }^{\mathrm{I}} /\left(1+x_{1, \exp }^{\mathrm{I}}\right)=0.0202$ and $y_{ \pm, \exp }^{\mathrm{II}}=x_{1, \exp }^{\mathrm{II}} /\left(1+x_{1, \exp }^{\mathrm{II}}\right)=0.3080$. For n-hexanol, $d_{2}=807 \mathrm{~kg} / \mathrm{m}^{3}$ and $\epsilon_{2}=10.7$ [29], giving $A_{\phi}=8.80$. In principle the closest approach parameter $\rho$ depends on the properties of the electrolyte and on how the shortrange forces are modeled. However, for simplicity in practice, it is generally taken to be a constant applicable to a wide variety of salts. In the development of the eNRTL model [14], a value of $\rho=14.9$ was used, as originally suggested by Pitzer [15], and was found to be satisfactory for a large number of aqueous inorganic electrolytes. However, since ILs such as $\left[\right.$ bmpy] $\left[\mathrm{Tf}_{2} \mathrm{~N}\right]$ involve bulky organic cations, a larger value of the closest approach parameter may be appropriate for this group of salts. For this purpose, we suggest here using the value $\rho=25$, though it may be found in subsequent work that some other value is more appropriate. In this problem, and in the remaining two examples involving ILs, we will do parameter estimation using both the "traditional" value of $\rho=14.9$ and the value of $\rho=25$. Both the NRTL and eNRTL models, with $\alpha=0.2$ in each case, will be used.

Applying the interval method to the solution of Eq. (3) for the NRTL model and Eq. 
(7) for the eNRTL model with $\rho=14.9$ and $\rho=25$, we find the parameter solutions shown in Table 5. Both of the NRTL parameter solutions represent stable LLE. However, solution 2 has one large negative parameter value which leads to an unrealistic Gibbs energy surface. Thus, solution 1 is the preferred solution and is the one presented by Crosthwaite et al. [27], who used the method presented here to do their parameter estimation. For phase stability analysis with the eNRTL model, we extended the interval-based global optimization method [6] used for NRTL. When eNRTL is used with $\rho=14.9$, there are four parameter solutions. However, two of these do not represent stable phase equilibrium, and the other two each have one large negative parameter value, making them unsuitable, as discussed above. Thus, this "traditional" value of $\rho$ does not appear to be appropriate. However, with $\rho=25$ there are three stable solutions not involving large negative parameter values. To judge the suitability of these three solutions we examine the Gibbs energy versus composition surface ( $g^{\mathrm{M}}$ vs. $\left.y_{ \pm}\right)$ as given by Eq. (10). These surfaces are shown in Figs. 1-3. On such a plot, liquid-liquid equilibrium is indicated by a line bitangent to the Gibbs energy curve, with the two points of tangency corresponding to the equilibrium phase compositions. The bitangent line shown in each figure represents the experimental LLE at $y_{ \pm, \exp }^{\mathrm{I}}=0.0202$ and $y_{ \pm, \exp }^{\mathrm{II}}=0.3080$. It can also be seen that in each case this is the only possible bitangent line. Thus, none of these solutions leads to a model predicting multiple immiscibility gaps. All three of the $g^{\mathrm{M}}$ vs. $y_{ \pm}$curves have only two inflection points. Based on the Gibbs energy curves, it is difficult to say that any one of the three parameter solutions is preferable; thus, the decision may depend on context. For example, if the parameter estimation is being done 
using mutual solubility data at multiple temperatures, then at each temperature there is a set of multiple parameter solutions, such as seen here, that may be obtained. The $\tau_{12}$ and $\tau_{21}$ parameters are generally found to vary linearly with temperature, so those solutions that provide the best linear correlation with temperature could be chosen. Another possibility is that the parameter estimation from binary data is being done as part of an effort to model multicomponent data. In this context, the parameter solution that provides the best match to multicomponent data could be chosen.

\section{$4.5 \quad[\mathrm{bmim}]\left[\mathrm{Tf}_{2} \mathrm{~N}\right]$ and $n$-Butanol}

Consider a mixture of 1-n-butyl-3-methylimidazolium bis(trifluoromethylsulfonyl)imide $\left([\mathrm{bmim}]\left[\mathrm{Tf}_{2} \mathrm{~N}\right]\right)(1)$ and $n$-butanol $(2)$ at $T=288 \mathrm{~K}$ at atmospheric pressure. This system has been studied experimentally by Najdanovic-Visak et al. [30], and from their cloud point

correlation the mutual solubility at $T=288 \mathrm{~K}$ is $x_{1, \exp }^{\mathrm{I}}=0.021460$ and $x_{1, \exp }^{\mathrm{II}}=0.39889$. For $n$-butanol, $d_{2}=810 \mathrm{~kg} / \mathrm{m}^{3}$ and $\epsilon_{2}=17.8$ [31], making $A_{\phi}=4.84$. Using both NRTL and eNRTL ( $\alpha=0.2$ in both cases), the interval method is used to solve for the parameters, with the results shown in Table 6.

Again the preferred NRTL solution is the first one, as the second solution has a large negative parameter value. For this system, eNRTL with $\rho=14.9$ provides several stable parameter solutions, including three with suitable parameter values. However, it provided no suitable parameter solutions in the previous example, and will provide none in the next example either. Since, as discussed above, it is desirable to be able to use the same value 
of $\rho$ for all the salts in these examples, we choose not to use any of these solutions. Using $\rho=25$ for this system yields one stable solution not involving a large negative parameter value, and that is the result (solution 1) we consider most appropriate.

\section{6 $[\mathrm{hmim}]\left[\mathrm{Tf}_{2} \mathrm{~N}\right]$ and $n$-Octanol}

In this final example we consider the system of 1-n-hexyl-3-methylimidazolium bis(trifluoromethylsulfonyl)imide $\left([\mathrm{hmim}]\left[\mathrm{Tf}_{2} \mathrm{~N}\right]\right)(1)$ and $n$-octanol (2) at $T=298.15 \mathrm{~K}$ and atmospheric pressure. The experimental mutual solubility data [32] is $x_{1, \exp }^{\mathrm{I}}=0.0100$ and $x_{1, \exp }^{\mathrm{II}}=0.6450$. For $n$-octanol, $d_{2}=821 \mathrm{~kg} / \mathrm{m}^{3}$ and $\epsilon_{2}=10.0[31]$, so $A_{\phi}=10.9$. Applying the interval method gives the parameter estimation results shown in Table 7 for NRTL and eNRTL (both using $\alpha=0.2$ ).

For NRTL, there is again one solution with a large negative parameter value that can be ruled out. For eNRTL with $\rho=14.9$ there are two stable solutions, but neither is suitable due to the large negative $\tau_{12}$ values. This result and the result for the $[\mathrm{bmpy}]\left[\mathrm{Tf}_{2} \mathrm{~N}\right]$ and $n$-hexanol system make it clear why we have chosen to employ a different value of $\rho$. For eNRTL with $\rho=25$, there are five stable solutions. Solutions 3,7 and 8 can be ruled out based on their each having a large negative parameter value. However, both solutions 1 and 2 are suitable parameter values and the choice of which to use may depend on context or other considerations, as discussed above. 


\section{Concluding Remarks}

We have described here the use of an interval method for determining parameter values from mutual solubility data in two-parameter models of liquid-liquid equilibrium. An interval-based global optimization method is used to determine if the parameter values correspond to stable LLE. The method is mathematically and computationally guaranteed to locate all sets of parameter values corresponding to stable phase equilibria. The technique was demonstrated with examples using the NRTL and electrolyte NRTL (eNRTL) models. In two of the NRTL examples, results were found that contradict previous work. In the eNRTL examples, binary systems of an ionic liquid and an alcohol were considered. This appears to be the first time that a method for parameter estimation in the eNRTL model from binary LLE data (mutual solubility) has been presented. While we have focused here on the NRTL and eNRTL models, this technique can be applied in connection with any two-parameter activity coefficient model for LLE in which there is the potential for multiple parameter solutions.

\section{Acknowledgements}

This work was supported in part by the Department of Education Graduate Assistance in Areas of National Needs (GAANN) Program under Grant \#P200A010448, by the State of Indiana 21st Century Research and Technology Fund under Grant \#909010455 and by the National Oceanic and Atmospheric Administration under Grant \#NA050AR4601153. 


\section{Appendix A. NRTL Model}

The NRTL model for a binary system is given by:

$$
\frac{g^{\mathrm{E}}}{R T}=x_{1} x_{2}\left(\frac{\tau_{21} G_{21}}{x_{1}+x_{2} G_{21}}+\frac{\tau_{12} G_{12}}{x_{2}+x_{1} G_{12}}\right),
$$

where $g^{\mathrm{E}}$ is the molar excess Gibbs energy, and

$$
\begin{gathered}
\tau_{12}=\frac{g_{12}-g_{22}}{R T}=\frac{\Delta g_{12}}{R T} \\
\tau_{21}=\frac{g_{21}-g_{11}}{R T}=\frac{\Delta g_{21}}{R T} \\
G_{12}=\exp \left(-\alpha_{12} \tau_{12}\right) \\
G_{21}=\exp \left(-\alpha_{21} \tau_{21}\right) .
\end{gathered}
$$

Here $g_{i j}$ is an energy parameter characteristic of the $i-j$ interaction, $R$ is the gas constant, $T$ is the absolute temperature, and the parameter $\alpha=\alpha_{12}=\alpha_{21}$ is related to the nonrandomness in the mixture. The activity coefficients corresponding to Eq. (A.1) are given by

$$
\begin{aligned}
& \ln \gamma_{1}=x_{2}^{2}\left[\tau_{21}\left(\frac{G_{21}}{x_{1}+x_{2} G_{21}}\right)^{2}+\frac{\tau_{12} G_{12}}{\left(x_{2}+x_{1} G_{12}\right)^{2}}\right] \\
& \ln \gamma_{2}=x_{1}^{2}\left[\tau_{12}\left(\frac{G_{12}}{x_{2}+x_{1} G_{12}}\right)^{2}+\frac{\tau_{21} G_{21}}{\left(x_{1}+x_{2} G_{21}\right)^{2}}\right] .
\end{aligned}
$$




\section{Appendix B. Electrolyte-NRTL Model}

When normalized for a symmetric reference state (pure dissociated liquid 1 and pure liquid 2, both at system temperature and pressure), the electrolyte-NRTL (eNRTL) model for a binary system is given by $[14,15]$ :

$$
\frac{g^{\mathrm{E}}}{R T}=\frac{g_{\mathrm{PDH}}}{R T}+\frac{g_{\mathrm{LC}}}{R T}
$$

where

$$
\begin{gathered}
\frac{g_{\mathrm{PDH}}}{R T}=-\frac{40}{\rho} \sqrt{\frac{10}{M_{2}}} A_{\phi} I_{y} \ln \left(\frac{1+\rho \sqrt{I_{y}}}{1+\rho \sqrt{I_{1}^{\circ}}}\right) \\
I_{y}=\frac{1}{2}\left(z_{+}^{2} y_{+}+z_{-}^{2} y_{-}\right)
\end{gathered}
$$

with $I_{1}^{\circ}=\lim _{x_{1} \rightarrow 1} I_{y}$ and

$$
\begin{aligned}
\frac{g_{\mathrm{LC}}}{R T}=y_{2}\left(\frac{\left(y_{+}+y_{-}\right) G_{12}}{\left(y_{+}+y_{-}\right) G_{12}+y_{2}}\right) \tau_{12}+y_{+} z_{+}\left(1-\frac{y_{-}}{y_{-}+y_{2} G_{21}}\right) \tau_{21} & \\
& +y_{-}\left|z_{-}\right|\left(1-\frac{y_{+}}{y_{+}+y_{2} G_{21}}\right) \tau_{21}
\end{aligned}
$$

with $G_{12}=\exp \left(-\alpha \tau_{12}\right)$ and $G_{21}=\exp \left(-\alpha \tau_{21}\right)$. Here $g_{\mathrm{PDH}}$ is the Pitzer extended DebyeHückel model for the long-range electrostatic contribution, $g_{\mathrm{LC}}$ is an NRTL-type local composition contribution, $M_{2}$ is the molecular weight of the solvent, $\rho$ is the "closest approach" parameter, and $A_{\phi}$ is the usual Debye-Hückel parameter defined as

$$
A_{\phi}=\frac{1}{3} \sqrt{\frac{2 \pi N_{A} d_{2}}{1000}}\left(\frac{e^{2}}{\epsilon_{0} \epsilon_{2} k T}\right)^{3 / 2} .
$$

Here $N_{A}$ is Avogadro's number, $d_{2}$ is the density of the solvent in $\mathrm{kg} / \mathrm{m}^{3}, e$ is the elementary

charge, $\epsilon_{0}$ is the permittivity of free space, $\epsilon_{2}$ is the dielectric constant of the solvent, $k$ is Boltzmann's constant, $R$ is the gas constant, and $T$ is the absolute temperature. 
Since the examples used here involve only 1:1 electrolytes $\left(\nu_{+}=\nu_{-}=1\right)$ with $z_{+}=1$ and $z_{-}=-1$, we show here the activity coefficient expressions only for this case:

$$
\begin{gathered}
\ln \gamma_{i}=\ln \gamma_{i}^{\mathrm{PDH}}+\ln \gamma_{i}^{\mathrm{LC}}, \quad i= \pm, 2 \\
\ln \gamma_{ \pm}^{\mathrm{PDH}}=-\sqrt{\frac{500}{M_{2}}} A_{\phi}\left[\frac{2^{3 / 2}}{\rho} \ln \left(\frac{1+\rho \sqrt{y_{ \pm}}}{1+\frac{\rho}{\sqrt{2}}}\right)+\frac{\left(2 y_{ \pm}\right)^{1 / 2}-\left(2 y_{ \pm}\right)^{3 / 2}}{1+\rho \sqrt{y_{ \pm}}}\right] \\
\ln \gamma_{ \pm}^{\mathrm{LC}}=\frac{y_{2}^{2} G_{12} \tau_{12}}{\left(2 y_{ \pm} G_{12}+y_{2}\right)^{2}}-\frac{y_{ \pm} y_{2} G_{21} \tau_{21}}{\left(y_{ \pm}+y_{2} G_{21}\right)^{2}}+\frac{y_{2} G_{21} \tau_{21}}{y_{ \pm}+y_{2} G_{21}} \\
\ln \gamma_{2}^{\mathrm{PDH}}=\frac{2 \sqrt{\frac{1000}{M_{2}}} A_{\phi} y_{ \pm}^{3 / 2}}{1+\rho \sqrt{y_{ \pm}}} \\
\ln \gamma_{2}^{\mathrm{LC}}=2\left(\frac{y_{ \pm}^{2} G_{21} \tau_{21}}{\left(y_{ \pm}+y_{2} G_{21}\right)^{2}}-\frac{y_{ \pm} y_{2} G_{12} \tau_{12}}{\left(2 y_{ \pm} G_{12}+y_{2}\right)^{2}}+\frac{y_{ \pm} G_{12} \tau_{12}}{2 y_{ \pm} G_{12}+y_{2}}\right) .
\end{gathered}
$$




\section{References}

[1] H. Renon, J. M. Prausnitz, AIChE J. 14 (1968) 135-144.

[2] H. Renon, J. Prausnitz, Ind. Eng. Chem. Proc. Des. Dev. 8 (1969) 413-419.

[3] J. M. Sørensen, W. Arlt, Liquid-Liquid Equilibrium Data Collection, Chemistry Data Series, Vol. V, Parts 1-3, DECHEMA, Frankfurt/Main, Germany, 1979-1980.

[4] J. P. Novák, J. Matouš, J. Pick, Liquid-Liquid Equilibria, Elsevier, New York, NY, 1987.

[5] M. A. Stadtherr, C. A. Schnepper, J. F. Brennecke, AIChE Symp. Ser. 91(304) (1995) $356-359$.

[6] S. R. Tessier, J. F. Brennecke, M. A. Stadtherr, Chem. Eng. Sci. 55 (2000) 1785-1796.

[7] L. E. Baker, A. C. Pierce, K. D. Luks, Soc. Petrol. Engrs. J. 22 (1982) 731-742.

[8] M. L. Michelsen, Fluid Phase Equilib. 9 (1982) 1-19.

[9] R. A. Heidemann, J. M. Mandhane, Chem. Eng. Sci. 28 (1973) 1213-1221.

[10] A. C. Mattelin, L. A. J. Verhoeye, Chem. Eng. Sci. 30 (1975) 193-200.

[11] J. Jacq, L. Asselineau, Fluid Phase Equilibria 14 (1983) 185-192.

[12] R. A. Robinson, R. H. Stokes, Electrolyte Solutions, 2nd Ed., Butterworths, London, UK, 1959. 
[13] H. Zerres, J. M. Prausnitz, AIChE J. 40 (1994) 676-691.

[14] C.-C. Chen, H. I. Britt, J. F. Boston, L. B. Evans, AIChE J. 28 (1982) 588-596.

[15] K. S. Pitzer, J. Am. Chem. Soc. 102 (1980) 2902-2906.

[16] C.-C. Chen, L. B. E. and, AIChE J. 32 (1986) 444-454.

[17] C.-C. Chen, Y. Song, AIChE J. 50 (2004) 1928-1941.

[18] L. S. Belvéze, J. F. Brennecke, M. A. Stadtherr, Ind. Eng. Chem. Res. 43 (2004) 815825.

[19] Y. Liu, S. Watanasiri, Fluid Phase Equilib. 116 (1996) 193-200.

[20] R. B. Kearfott, Rigorous Global Search: Continuous Problems, Kluwer Academic Publishers, Dordrecht, The Netherlands, 1996.

[21] A. Neumaier, Interval Methods for Systems of Equations, Cambridge University Press, Cambridge, UK, 1990.

[22] E. Hansen, G. W. Walster, Global Optimization Using Interval Analysis, Marcel Dekker, New York, NY, 2004.

[23] C. A. Schnepper, M. A. Stadtherr, Comput. Chem. Eng. 20 (1996) 187-199.

[24] C.-Y. Gau, M. A. Stadtherr, Comput. Chem. Eng. 26 (2002) 827-840.

[25] Y. Lin, M. A. Stadtherr, Ind. Eng. Chem. Res. 43 (2004) 3741-3749. 
[26] G. Xu, A. M. Scurto, M. Castier, J. F. Brennecke, M. A. Stadtherr, Ind. Eng. Chem. Res. 39 (2000) 1624-1636.

[27] J. M. Crosthwaite, M. J. Muldoon, S. N. V. K. Aki, E. J. Maginn, J. F. Brennecke, J. Phys. Chem. B 110 (2006) 9354-9361.

[28] A. E. Ayala, L. D. Simoni, Y. Lin, J. F. Brennecke, M. A. Stadtherr, Computer-Aided Chemical Engineering 21 (2006) 463-468.

[29] R. P. Singh, C. P. Sinha, J. Chem. Eng. Data 27 (1982) 283-287.

[30] V. Najdanovic-Visak, L. P. N. Rebelo, M. N. da Ponte, Green Chem. 7 (2005) 443-450.

[31] C. P. Smyth, W. N. Stoops, J. Am. Chem. Soc. 51 (1929) 3312-3329.

[32] J. M. Crosthwaite, S. N. V. K. Aki, E. J. Maginn, J. F. Brennecke, J. Phys. Chem. B 108 (2004) 5113-5119. 
Table 1: NRTL parameter solutions for the mixture $n$-octanol and water $(\alpha=0.20, T=$ 313.15 K). $\Delta g_{12}$ and $\Delta g_{21}$ are given in $\mathrm{J} / \mathrm{mol}$.

\begin{tabular}{cccccc}
\hline Solution & $\Delta g_{12}$ & $\Delta g_{21}$ & $\tau_{12}$ & $\tau_{21}$ & Stable? \\
\hline 1 & 99.520 & 22304 & 0.038225 & 8.5668 & Yes \\
2 & 58860 & 22356 & 22.608 & 8.5868 & Yes \\
3 & 14293 & 120780 & 5.4922 & 46.392 & No \\
4 & 12784 & 120880 & 4.9101 & 46.428 & No
\end{tabular}


Table 2: Comparison of NRTL parameter estimates for the mixture $n$-butanol and water ( $\alpha=0.4, T=363 \mathrm{~K}$ ) obtained by Heidemann and Mandhane [9] and by the use of the interval method.

\begin{tabular}{cllc|lcc} 
& \multicolumn{4}{c}{ Heidemann and Mandhane [9] } & \multicolumn{3}{c}{ Interval Method } \\
\hline Solution & $\tau_{12}$ & $\tau_{21}$ & Stable? & $\tau_{12}$ & $\tau_{21}$ & Stable? \\
\hline 1 & 0.0074518 & 3.8021 & Yes & 0.0074518 & 3.8021 & Yes \\
2 & 10.182 & 3.8034 & Yes & 10.178 & 3.8034 & Yes \\
3 & -73.824 & -15.822 & Yes & & & \\
\hline
\end{tabular}


Table 3: NRTL parameter estimates for the mixture 1,4-dioxane and 1,2,3-propanetriol $(T=298.15 \mathrm{~K})$ found using the interval method for $\alpha=0.15$ and $\alpha=0.25$. Mattelin and Verhoeye [10] reported finding 6 solutions for $\alpha=0.15$ and 3 solutions for $\alpha=0.25$.

\begin{tabular}{cllc|lcc}
\multicolumn{4}{c}{$\alpha=0.15$} & \multicolumn{5}{c}{$\alpha=0.25$} \\
\hline Solution & $\tau_{12}$ & $\tau_{21}$ & Stable? & $\tau_{12}$ & $\tau_{21}$ & Stable? \\
\hline 1 & 5.6379 & -0.59940 & Yes & 4.5512 & 0.54810 & Yes \\
2 & 13.478 & -82.941 & Yes & 4.8352 & 11.826 & No \\
3 & 38.642 & 13.554 & No & & & \\
4 & 39.840 & 3.0285 & No & & &
\end{tabular}


Table 4: Comparison of number of solutions for the mixture 1,4-dioxane and 1,2,3propanetriol $(T=298.15 \mathrm{~K})$ found by Mattelin and Verhoeye [10] and by the interval method (IN/GB) for several values of $\alpha$.

\begin{tabular}{r|ccccccccccccc}
$\alpha$ & 0.05 & 0.076 & 0.10 & 0.125 & 0.15 & 0.175 & 0.20 & 0.25 & 0.3 & 0.35 & 0.40 & 0.427 \\
\hline$[10]$ & 6 & 5 & 4 & 5 & 6 & 5 & 4 & 3 & 2 & 2 & 2 & 1 \\
IN/GB & 4 & 4 & 4 & 4 & 4 & 4 & 4 & 2 & 2 & 2 & 0 & 0
\end{tabular}


Table 5: NRTL and eNRTL $(\rho=14.9$ and 25$)$ parameter solutions for $[\mathrm{bmpy}]\left[\mathrm{Tf}_{2} \mathrm{~N}\right]$ and $n$-hexanol $(T=321 \mathrm{~K}, \alpha=0.2)$.

\begin{tabular}{|c|c|c|c|c|c|c|c|c|c|}
\hline & & NRTL & & $\mathrm{eNI}$ & $\mathrm{TL}(\rho=1$ & 1.9) & $\mathrm{eN}$ & $\mathrm{RL}(\rho=2$ & \\
\hline Solution & $\tau_{12}$ & $\tau_{21}$ & Stable? & $\tau_{12}$ & $\tau_{21}$ & Stable? & $\tau_{12}$ & $\tau_{21}$ & Stable? \\
\hline 1 & -1.2378 & 5.2541 & Yes & -3.2193 & 5.2049 & No & -2.8136 & 5.6214 & Yes \\
\hline 2 & -50.427 & 12.997 & Yes & -2.4192 & 3.1407 & No & 0.012733 & 0.78331 & Yes \\
\hline 3 & & & & -47.899 & 25.965 & Yes & 3.5240 & -0.96382 & Yes \\
\hline 4 & & & & -52.946 & 0.032378 & Yes & 6.1094 & -22.605 & Yes \\
\hline 5 & & & & & & & 10.164 & 32.594 & No \\
\hline 6 & & & & & & & -42.019 & 17.882 & Yes \\
\hline
\end{tabular}


Table 6: NRTL and eNRTL $(\rho=14.9$ and 25$)$ parameter solutions for $[\mathrm{bmim}]\left[\mathrm{Tf}_{2} \mathrm{~N}\right]$ and $n$-butanol $(T=288 \mathrm{~K}, \alpha=0.2)$.

\begin{tabular}{|c|c|c|c|c|c|c|c|c|c|}
\hline & \multicolumn{3}{|c|}{ NRTL } & \multicolumn{3}{|c|}{$\operatorname{eNRTL}(\rho=14.9)$} & \multicolumn{3}{|c|}{$\operatorname{eNRTL}(\rho=25)$} \\
\hline Solution & $\tau_{12}$ & $\tau_{21}$ & Stable? & $\tau_{12}$ & $\tau_{21}$ & Stable? & $\tau_{12}$ & $\tau_{21}$ & Stable? \\
\hline 1 & -1.4245 & 5.4372 & Yes & -2.9302 & 5.8533 & Yes & -2.6641 & 6.1543 & Yes \\
\hline 2 & -50.094 & 13.626 & Yes & -0.019516 & 0.73181 & Yes & 1.7764 & 29.129 & No \\
\hline 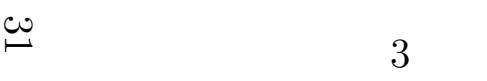 & & & & 3.7634 & -1.0776 & Yes & 7.4641 & 27.916 & No \\
\hline 4 & & & & 6.3820 & -22.004 & Yes & -41.827 & 16.838 & Yes \\
\hline 5 & & & & 23.712 & 0.70331 & No & & & \\
\hline 6 & & & & 10.154 & 33.433 & No & & & \\
\hline 7 & & & & -42.348 & 18.725 & Yes & & & \\
\hline 8 & & & & -84.504 & 0.71319 & Yes & & & \\
\hline
\end{tabular}


Table 7: NRTL and eNRTL $(\rho=14.9$ and 25$)$ parameter solutions for $[\mathrm{hmim}]\left[\mathrm{Tf}_{2} \mathrm{~N}\right]$ and $n$-octanol $(T=298 \mathrm{~K}, \alpha=0.2)$.

\begin{tabular}{|c|c|c|c|c|c|c|c|c|c|}
\hline & & NRTL & & eNF & $\Gamma L(\rho=$ & 4.9) & eNF & $\mathrm{TL}(\rho=$ & \\
\hline Solution & $\tau_{12}$ & $\tau_{21}$ & Stable? & $\tau_{12}$ & $\tau_{21}$ & Stable? & $\tau_{12}$ & $\tau_{21}$ & Stable? \\
\hline 1 & -0.57053 & 5.1401 & Yes & -48.046 & 22.107 & Yes & -0.94386 & 1.7226 & Yes \\
\hline 2 & -58.495 & 10.500 & Yes & -57.265 & 0.19151 & Yes & -2.5081 & 4.6644 & Yes \\
\hline 3 & & & & & & & 9.9928 & -30.466 & Yes \\
\hline 4 & & & & & & & 5.5007 & -1.1504 & No \\
\hline 5 & & & & & & & 14.547 & 0.29352 & No \\
\hline 6 & & & & & & & 12.677 & 37.640 & No \\
\hline 7 & & & & & & & -46.975 & 17.085 & Yes \\
\hline 8 & & & & & & & -67.518 & 0.82012 & Yes \\
\hline
\end{tabular}




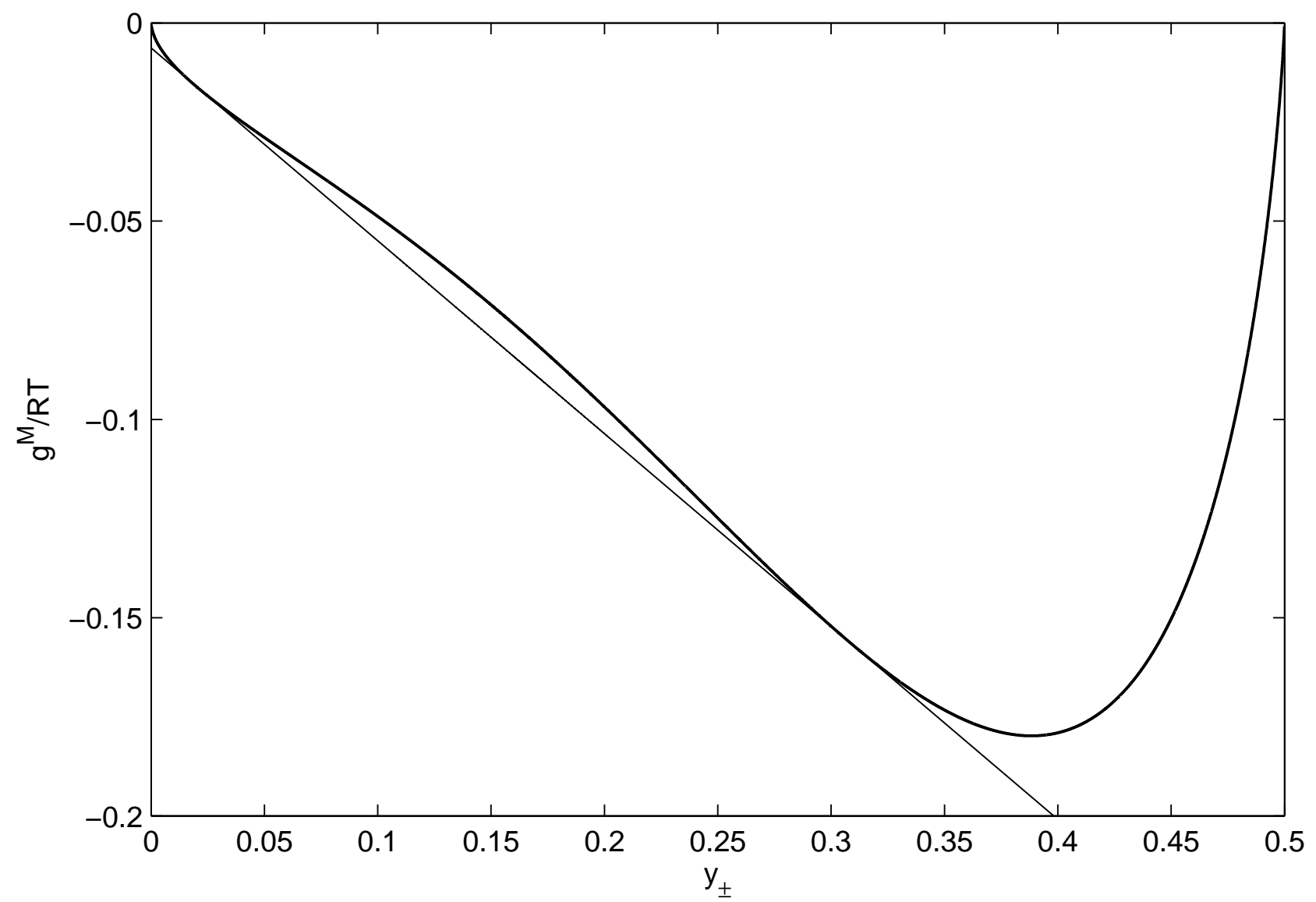

Figure 1: Gibbs energy versus composition surface for eNRTL $(\rho=25)$ with parameter solution 1 (see Table 5) for the $[\mathrm{bmpy}]\left[\mathrm{Tf}_{2} \mathrm{~N}\right]$ and $n$-hexanol system. The bitangent line represents the experimental LLE at $y_{ \pm, \exp }^{\mathrm{I}}=0.0202$ and $y_{ \pm, \exp }^{\mathrm{II}}=0.3080$. 


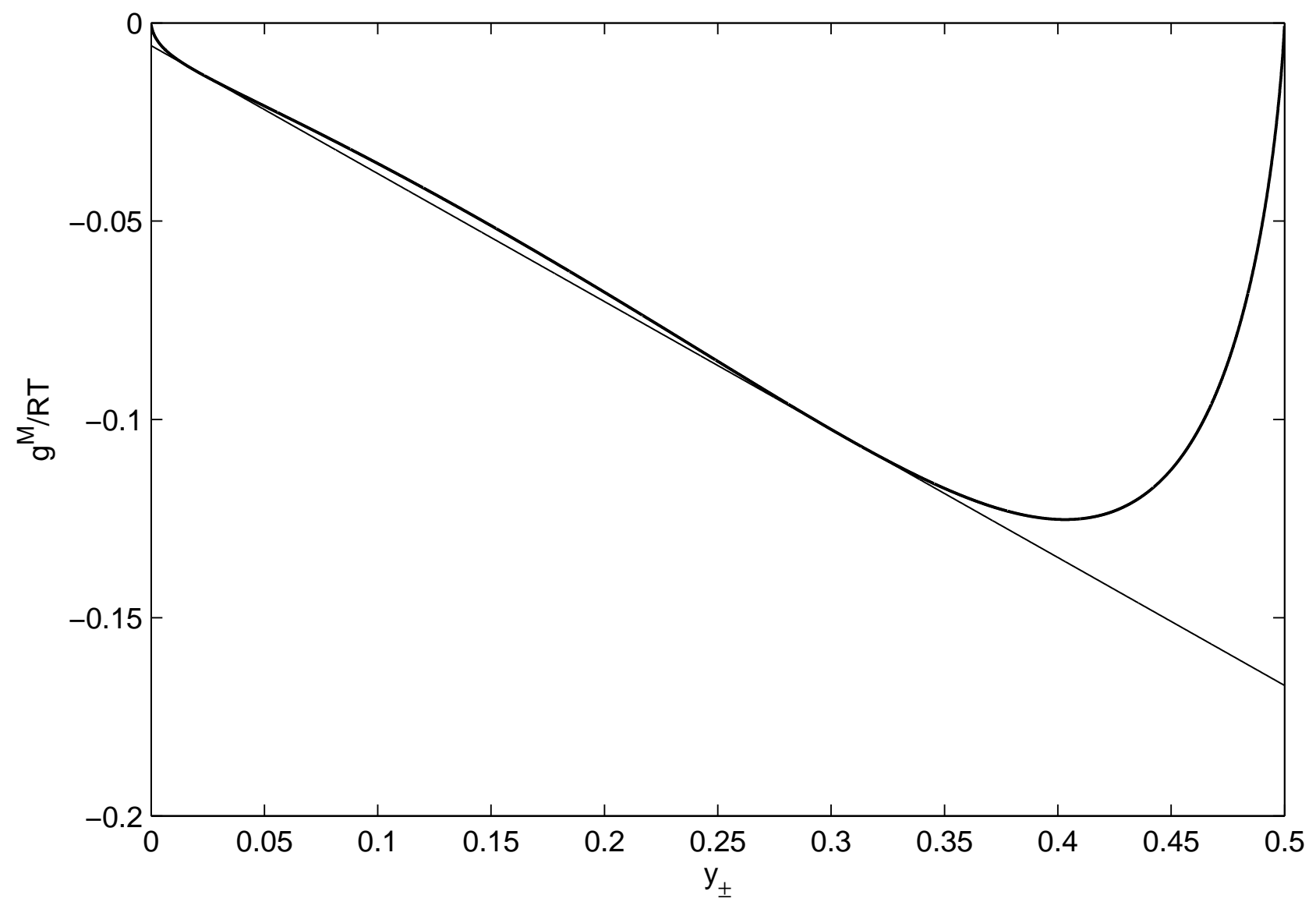

Figure 2: Gibbs energy versus composition surface for eNRTL $(\rho=25)$ with parameter solution 2 (see Table 5) for the $[\mathrm{bmpy}]\left[\mathrm{Tf}_{2} \mathrm{~N}\right]$ and $n$-hexanol system. The bitangent line represents the experimental LLE at $y_{ \pm, \exp }^{\mathrm{I}}=0.0202$ and $y_{ \pm, \exp }^{\mathrm{II}}=0.3080$. 


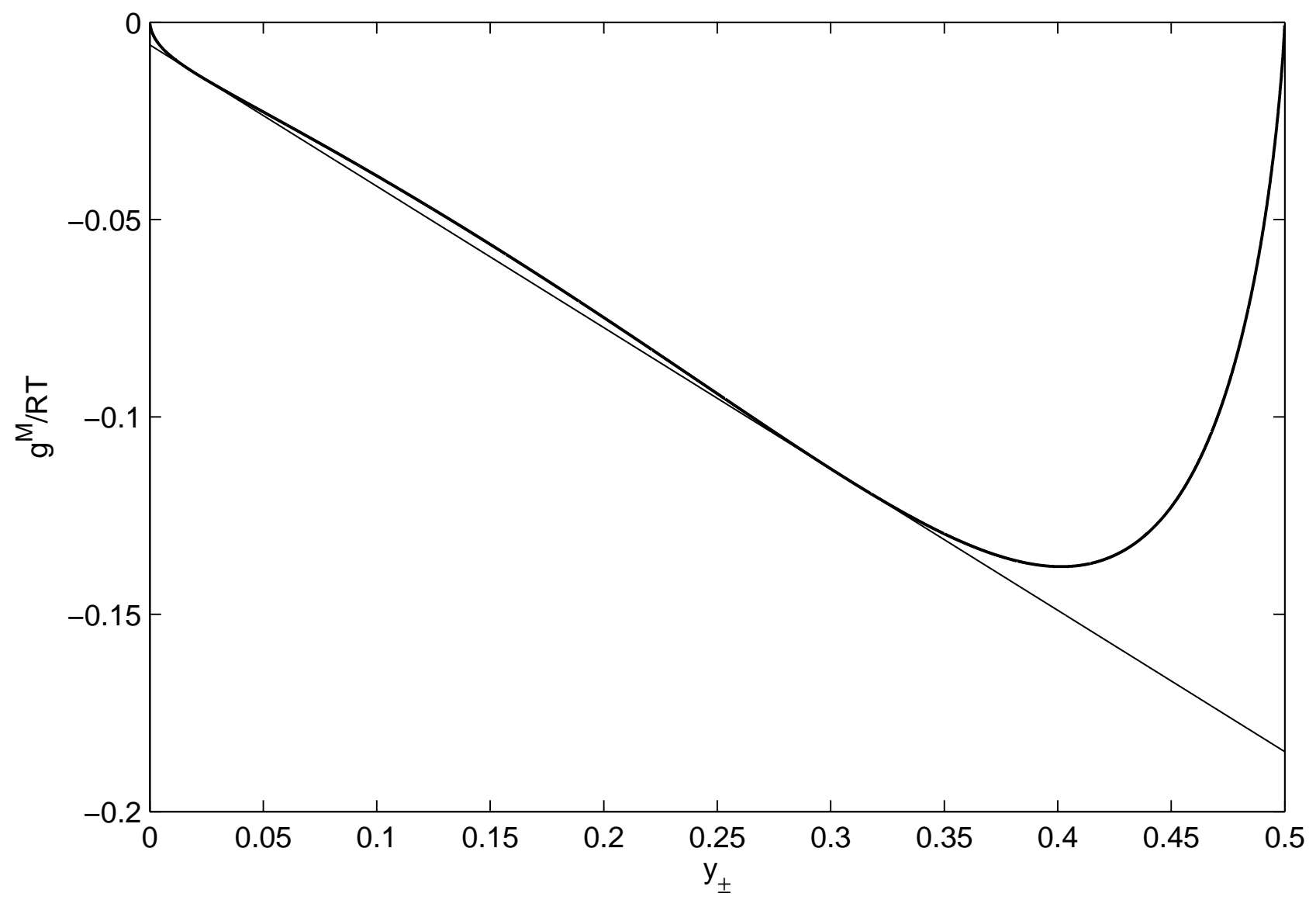

Figure 3: Gibbs energy versus composition surface for eNRTL $(\rho=25)$ with parameter solution 3 (see Table 5) for the $[\mathrm{bmpy}]\left[\mathrm{Tf}_{2} \mathrm{~N}\right]$ and $n$-hexanol system. The bitangent line represents the experimental LLE at $y_{ \pm, \exp }^{\mathrm{I}}=0.0202$ and $y_{ \pm, \exp }^{\mathrm{II}}=0.3080$. 